Service social

\title{
Mothers on Trial - The Battle for Children and Custody, par Phyllis Chesler, Seattle, Seal Press, 1986, 651 pages.
}

\section{Francine Lavoie}

Volume 37, numéro 1-2, 1988

Par-delà les barrières des sexes

URI : https://id.erudit.org/iderudit/706399ar

DOI : https://doi.org/10.7202/706399ar

Aller au sommaire du numéro

Éditeur(s)

École de service social de l'Université Laval

ISSN

1708-1734 (numérique)

Découvrir la revue

Citer ce compte rendu

Lavoie, F. (1988). Compte rendu de [Mothers on Trial - The Battle for Children and Custody, par Phyllis Chesler, Seattle, Seal Press, 1986, 651 pages.] Service social, 37(1-2), 273-275. https://doi.org/10.7202/706399ar d'utilisation que vous pouvez consulter en ligne.

https://apropos.erudit.org/fr/usagers/politique-dutilisation/ 
Mothers on Trial - The Battle for Children and Custody, par Phyllis CHESLER, Seattle, Seal Press, 1986, 651 pages.

Ce livre ne peut laisser indifférent. Il fait comprendre la vulnérabilité de toutes les mères, fait voir l'horreur de certaines décisions légales concernant la garde des enfants lors d'une séparation et fait réfléchir à notre tendance à survaloriser le père qui obtient la garde alors qu'on dévalorise la mère qui ne l'a pas. Ce livre ne peut que toucher. Par ailleurs, il peut agacer. Le mode de présentation de la méthode de recherche n'est pas au-dessus de tout soupçon. II s'agit d'un sympathique fouillis mais d'un fouillis tout de même. Mais décrivons d'abord le propos.

Phyllis Chesler veut faire comprendre ce que vivent "d'assez bonnes" mères lorsque leur conjoint livre bataille pour obtenir la garde des enfants. Il ne s'agit pas ici d'histoires de divorces idéaux, de garde partagée idyllique, ni d'histoires de pères compétents ou de mères incompétentes. II s'agit plutôt d'illustrer que, dans notre société, on reconnaît à l'homme des droits de garde de ses enfants quel que soit son comportement (incestueux, violent, négligent), alors qu'on n'attribue aucun droit à la mère, même responsable et compétente. Il faut rappeler que la majorité des mères ont effectivement la garde des enfants. Le phénomène étudié par Chesler touche une minorité de couples où, lorsque l'homme l'exige, il obtient la garde même si la mère est une bonne mère. Dans l'enquête menée par Chesler, $82 \%$ des mères ainsi contestées par le père ont perdu la garde. De 1975 à 1985, aux États-Unis, au moins deux millions de pères auraient obtenu la garde en kidnappant l'enfant, et $\mathbf{4 0 0} 000$ autres, en passant en Cour.

L'auteure s'interroge sur les raisons pouvant expliquer qu'on enlève ainsi tout droit à la mère alors qu'on ne peut lui reprocher d'être une "mauvaise" mère.

Grâce à un relevé de jugements de Cour ou de dossiers psychiatriques, on apprend que les raisons évoquées sont que la femme :

- a un revenu inférieur à celui de son ex-mari,

- ne peut faire vivre ses enfants avec son salaire,

- n'a pas d'heures de travail flexibles garantissant sa présence auprès des enfants,

- a une carrière professionnelle, ce qui montre qu'elle ne s'intéresse qu'à elle-même,

- n'a pas de carrière ou de métier,

- fait garder ses enfants pour aller travailler,

- a ou a eu une liaison, avant ou après le divorce,

- a des activités politiques offensant l'idéologie de son ex-mari,

- est reconnue comme lesbienne,

- a un autre enfant illégitime,

- désire changer de ville.

L'auteure souligne fort habilement que le fait que le père ait lui aussi quelques-unes de ces caractéristiques (liaison, faire garder les enfants, etc.) n'a pas la même incidence sur la décision légale. De plus, il est clairement montré 
que toutes les femmes sont vulnérables face à ce jugement (instruites ou pas, ayant une carrière ou pas). C'est la force du présent document de montrer que toutes les femmes pourraient vivre un tel drame.

Une série d'entrevues auprès de soixante femmes blanches scolarisées (niveau collégial) ayant vécu cette expérience entre 1960 et 1981, montre en effet que $82 \%$ d'entre elles ont perdu la garde. On apprend que le litige est survenu en moyenne après neuf ans de mariage, qu'il a duré environ trois ans et que, phénomène intéressant, les pères demandent la garde d'enfants propres, d'âge scolaire. Par ailleurs, le fait pour le père de kidnapper l'enfant ne nuit pas à l'obtention de la garde légale par la suite. L'auteure fournit un portrait des pères impliqués dans un tel litige : $62 \%$ ont été physiquement violents envers leur conjointe (avant ou pendant le litige), 33\% ont kidnappé l'enfant, $57 \%$ font subir à l'enfant un lavage de cerveau anti-mère, $50 \%$ se remarient ou co-habitent, $90 \%$ n'ont jamais payé de pension. Pendant le mariage, seulement $13 \%$ s'impliquaient dans les tâches d'éducation des enfants alors que $20 \%$ offraient une aide occasionnelle. Il ne s'agit donc pas du " super-père ". Ces données sont confirmées auprès d'un échantillon de femmes d'origines ethniques diverses.

D'autres questions sont abordées dans cet ouvrage : quel effet cette perte a-t-elle sur la vie des mères ? Quelles sont les motivations de ces pères? Un fois la bataille légale terminée, quel lien le père laisse-t-il se créer entre la mère et les enfants? Des comparaisons avec des femmes d'autres pays, avec des pères et des mères qui n'ont pas la garde mais qui acceptent ce fait, avec des mères en prison, avec des mères de bébés-éprouvettes permettent d'enrichir la discussion.

En ce qui a trait à la méthode de recherche, elle s'apparente à un collage de méthodes. Essentiellement multidimensionnelle, elle allie quelques statistiques à des analyses de contenu d'entrevues provenant de six enquêtes, à des citations d'œuvres littéraires ou d'ouvrages historiques, au dépouillement de dossiers. Les défauts d'une telle entreprise portent surtout sur la généralisation de telles données. Quelquefois l'auteure affirme que certains faits, constatés aux ÉtatsUnis, se déroulent également au Canada ou dans d'autres pays, et cela, sans avoir fouillé les dossiers ou mené une enquête en ce sens. De plus, elle tient peu compte de l'influence de l'histoire en amalgamant des cas datant de 1960 à 1981, ce qui semble fort critiquable. Elle tente de tenir compte de diverses classes sociales et de diverses origines ethniques mais, si elle rend bien les problèmes, elle tient peu compte du contexte social. Le traitement des données statistiques est quelquefois obscur. En fait, on perd le fil et, à certains moments, on ne sait plus de qui l'auteure parle.

Les qualités de cette approche pamphlétaire sont de suggérer mille pistes d'analyse de ce phénomène, d'allier, aux chiffres froids, des paroles de femmes touchées par ce problème, de faire une très large place à la discussion des "causes » du comportement des pères et à la remise en question de la société. La présence de données empiriques donne encore plus de poids à la réflexion de Chesler.

L'essentiel du message qu'elle veut livrer à la suite de ses enquêtes est que :

"Toutes les mères sont vulnérables au plan de la garde des enfants parce qu'elles sont des femmes; tous les pères, qu'ils soient incestueux, violents, 
absents, passifs ou "aidants", peuvent gagner la garde, non parce que les mères sont inadéquates ou que les pères sont des parents vraiment égaux, mais parce qu'ils sont des hommes. 》 (p. 394).

Un ouvrage à lire absolument par les intervenantes et intervenants, les juges, etc. Le Québec n'est sûrement pas épargné, mon expérience personnelle m'ayant déjà mis en contact avec une scientifique à qui on avait retiré la garde de ses enfants parce qu'elle travaillait!

Francine LAVOIE

École de psychologie,

Université Laval.

Un amour de père, par le collectif Cœur-Atout, Montréal, Saint-Martin, 1987, 238 pages.

Un amour de livre que cet Amour de père!

Mais situons d'abord les auteurs et le contexte. Le collectif Cœur-Atout est formé surtout d'ex-membres du groupe qui a été à la barre du bateau, un peu intrépide pour l'époque, qui avait pour nom Hom-Info. Revue sur la condition masculine publiée à Montréal durant plus de cinq ans, elle a dû tristement disparaître en 1985, faute d'argent. Les couleurs et du groupe et du livre s'en trouvent alors toutes révélées. Résolument consacré à la condition masculine, le collectif a organisé en juin 1986 sa première grande activité, le colloque national sur L'intervention auprès des hommes. ${ }^{1}$ Fort de ce succès, il répéta l'expérience en juin 1987 avec le colloque sur La part du père. Un amour de père en constitue les actes. Le livre présente un bon aperçu sur l'état de la condition paternelle d'ici. Réalité troublée et troublante, s'il en est, la paternité s'y trouve soumise avec soin au stéthoscope psychosocial, sans panique, mais sans complaisance. Même si ce n'est pas ici le lieu de les présenter toutes, le livre fourmille d'idées pas forcément neuves, mais nouvellement formulées souvent.

D'abord celles développées par Jean Monbourquette. Signataire de deux chapitres, il présente quelques réflexions dignes d'arrêt. Il affirme notamment le rôle essentiel des groupes d'hommes dans la démarche nécessaire de " masculinisation " des hommes : " ces groupes d'hommes [...] forment un nouvel utérus qui engendre le jeune homme à une masculinité pleine et plus affermie " (p. 156). Pour paraître abusive et complaisante, la position se comprend mieux quand on l'inscrit dans le contexte psychanalytique où la situe l'auteur. Une question alors : comment s'explique l'évolution de tortue qui caractérise partout les groupes d'hommes s'il faut y voir quelque chose d'aussi essentiel ? N'est-ce pas, par contre, réussir à mettre en mots cette espèce de climat, d'esprit, très particulier que reconnaît généralement à ces groupes tout participant qui s'y est engagé à fond ? À propos de l'évolution sociale qu'on a vécue, dans la foulée du féminisme, sur le plan des rapports entre les sexes, il prétend qu'elle s'est 